\title{
Additive value of $3 T$ cardiovascular magnetic resonance coronary angiography for detecting coronary artery disease
}

\author{
Lijun Zhang ${ }^{1}$, Xiantao Song ${ }^{2}$, Li Dong ${ }^{1}$, Jianan Li ${ }^{2}$, Ruiyu Dou', Zhanming Fan ${ }^{1 *}$, Jing $\mathrm{An}^{3}$ and Debiao Li ${ }^{4}$
}

\begin{abstract}
Background: The purpose of the work was to evaluate the incremental diagnostic value of free-breathing, contrastenhanced, whole-heart, $3 \mathrm{~T}$ cardiovascular magnetic resonance coronary angiography (CE-MRCA) to stress/rest myocardial perfusion imaging (MPI) and late gadolinium enhancement (LGE) imaging for detecting coronary artery disease (CAD).

Methods: Fifty-one patients with suspected CAD underwent a comprehensive cardiovascular magnetic resonance (CMR) examination (CE-MRCA, MPI, and LGE). The additive diagnostic value of MRCA to MPI and LGE was evaluated using invasive $x$-ray coronary angiography $(X A)$ as the standard for defining functionally significant $C A D(\geq 50 \%$ stenosis in vessels $>2 \mathrm{~mm}$ in diameter).

Results: $90.2 \%$ (46/51) patients ( $54.0 \pm 11.5$ years; $71.7 \%$ men) completed CE-MRCA successfully. On per-patient basis, compared to MPI/LGE alone or MPI alone, the addition of MRCA resulted in higher sensitivity (100\% vs. $76.5 \%, p<0.01$ ), no change in specificity ( $58.3 \%$ vs. $66.7 \%, p=0.6)$, and higher accuracy $(89.1 \%$ vs $73.9 \%, p<0.01)$ for CAD detection (prevalence $=73.9 \%$ ). Compared to LGE alone, the addition of CE-MRCA resulted in higher sensitivity $(97.1 \%$ vs. $41.2 \%$, $p<0.01$ ), inferior specificity (83.3\% vs. 91.7\%, $p=0.02)$, and higher diagnostic accuracy $(93.5 \%$ vs. $54.3 \%, p<0.01)$.

Conclusion: The inclusion of successful free-breathing, whole-heart, 3 T CE-MRCA significantly improved the sensitivity and diagnostic accuracy as compared to MPI and LGE alone for CAD detection.

Keywords: 3 Tesla, Contrast enhanced, Coronary magnetic resonance angiography, Stress-rest perfusion imaging, Late gadolinium enhancement, Coronary artery disease
\end{abstract}

\section{Background}

Coronary artery disease (CAD) is a leading cause of mortality and morbidity around the world $[1,2]$. Accurate diagnosis of CAD is important in risk stratification and in guiding clinical management [3-5]. Cardiovascular magnetic resonance (CMR) has emerged as an effective tool for the detection of CAD. CMR at both $1.5 \mathrm{~T}$ and $3 \mathrm{~T}$ has increasingly been used in clinical routine to assess myocardial ischemia and infarction caused by coronary artery stenosis using stress-rest myocardial perfusion imaging (MPI) and late gadolinium enhancement (LGE) imaging [6]. However, it is highly desirable to directly visualize coronary artery stenoses. CMR coronary angiography

\footnotetext{
* Correspondence: fanzm120@126.com

${ }^{1}$ Department of Radiology, Beijing Anzhen Hospital, Capital Medical University, Anzhenli Avenue, Chao Yang District, Beijing 100029, China Full list of author information is available at the end of the article
}

(MRCA) is technically challenging but has seen steady improvement in the last decade with highly promising clinical results [7-11]. At $1.5 \mathrm{~T}$, several studies have shown the incremental value of MRCA when added to CMR MPI and LGE $[12,13]$. The combined use of MRCA and CMR stress perfusion improved specificity for the detection of significant CAD [12]. Contrast-enhanced MRCA (CE-MRCA) at $3 \mathrm{~T}$ has shown excellent results in preliminary patient studies [10]. The purpose of this study was to evaluate the incremental value of CE-MRCA for the detection of CAD when added to routine CMR on $3 \mathrm{~T}$.

\footnotetext{
Methods

Patient population

From October 2015 to February 2017, consecutive patients with suspected CAD were prospectively recruited. Inclusion criteria were: (1) age 30 years or older, (2)
} 
presence of at least one major cardiovascular risk factor (smoking, diabetes, hypertension, or dyslipidemia), (3) documented stable angina. Figure 1 summarizes the study flow chart and the reasons for the exclusion of patients.

The study protocol was approved by the Ethics Committee of Beijing Anzhen Hospital. Written informed consent was obtained from the participants for publication of their individual details and images in this manuscript. The consent forms are held at the authors' institution and are available for review by the Editor-in-Chief.

\section{CMR protocol}

All examinations were performed on a $3 \mathrm{~T}$ whole-body scanner (MAGNETOM Verio, A Tim System; Siemens Healthineers, Erlangen, Germany) with a 32-element matrix coil. Detailed protocols with CE-MRCA and CMR are given in Fig. 2.

For CE-MRCA, a navigator-gated, electrocardiogram (ECG)-triggered, fat-saturated, inversion-recovery prepared segmented 3D gradient-echo sequence was employed [10]. Before examination, patients were trained to perform regular, shallow breathing and to avoid changes in depth of breathing during data acquisition [10]. Sixty seconds after the initiation of contrast agent administration (Magnevist, Bayer Healthcare,Berlin, Germany; $0.1 \mathrm{mmol} / \mathrm{kg}, 0.2 \mathrm{ml} / \mathrm{sec}$ ), whole-heart CEMRCA data acquisition was started. The imaging volume was prescribed in the axial plane to cover the entire heart. Prospective real-time adaptive motion correction was applied in the superior-inferior direction to compensate the respiratory motion based on the navigator signal with a correction factor of 0.6 [10]. Imaging parameters included: $\mathrm{TR} / \mathrm{TE}=3.0 / 1.4 \mathrm{~ms}$, flip angle $=20^{\circ}$, readout bandwidth $=610 \mathrm{~Hz} /$ pixel, acquired voxel size $=$ $1.3 \times 1.3 \times 1.3 \mathrm{~mm}^{3}$ and interpolated to $0.65 \times 0.65 \times 0$. $65 \mathrm{~mm}^{3}$. Data acquisition was accelerated by employing generalized autocalibrating partially parallel acquisitions (GRAPPA) in the phase-encoding direction with a factor of 2. A non-selective inversion pulse was applied prior to the navigator gating and data acquisition to suppress background tissues. The inversion recovery time (TI) was 200 msec.

For CMR MPI, a T1-weighted saturation-recovery fast gradient echo sequence was used with $\mathrm{TR} / \mathrm{TE}=165.0 / 1$. $1 \mathrm{~ms}$, flip angle $=12^{\circ}, \mathrm{TI}=100 \mathrm{~ms}, \quad \mathrm{FOV}=350 \times$ $450 \mathrm{~mm}^{2}$, slice thickness $=8 \mathrm{~mm}$. Three left ventricular (LV) short-axis slices (basal, midventricular, and apical) were acquired under maximal hyperemia achieved with $140 \mu \mathrm{g} / \mathrm{kg} / \mathrm{min}$ IV adenosine infusion for $5 \mathrm{~min}$, during the first pass of a bolus of $0.05 \mathrm{mmol} / \mathrm{kg}$ of contrast media (Magnevist, Bayer Healthcare) injected at $5 \mathrm{ml} / \mathrm{s}$. During the examination, blood pressure and ECG were continuously recorded. Ten minutes after the stress perfusion, the same scan was repeated at rest.

For LGE imaging, a 2D phase-sensitive inversion recovery breath-hold sequence was used at least $10 \mathrm{~min}$ after the last administration of gadolinium $(\mathrm{TR} / \mathrm{TE}=4.1$ / $1.56 \mathrm{~ms}$, flip angle $=20^{\circ}, \mathrm{FOV}=350 \times 284 \mathrm{~mm}^{2}$, slice thickness $=8 \mathrm{~mm}$ ). LGE images were acquired in two LV long-axis (two-chamber and four-chamber) views and in multiple short-axis views with a slice distance of $8 \mathrm{~mm}$, covering the whole LV from base to apex.

\section{Imaging analysis}

CE-MRCA was assessed by 2 experienced readers who were blinded to the patient information. Axial source

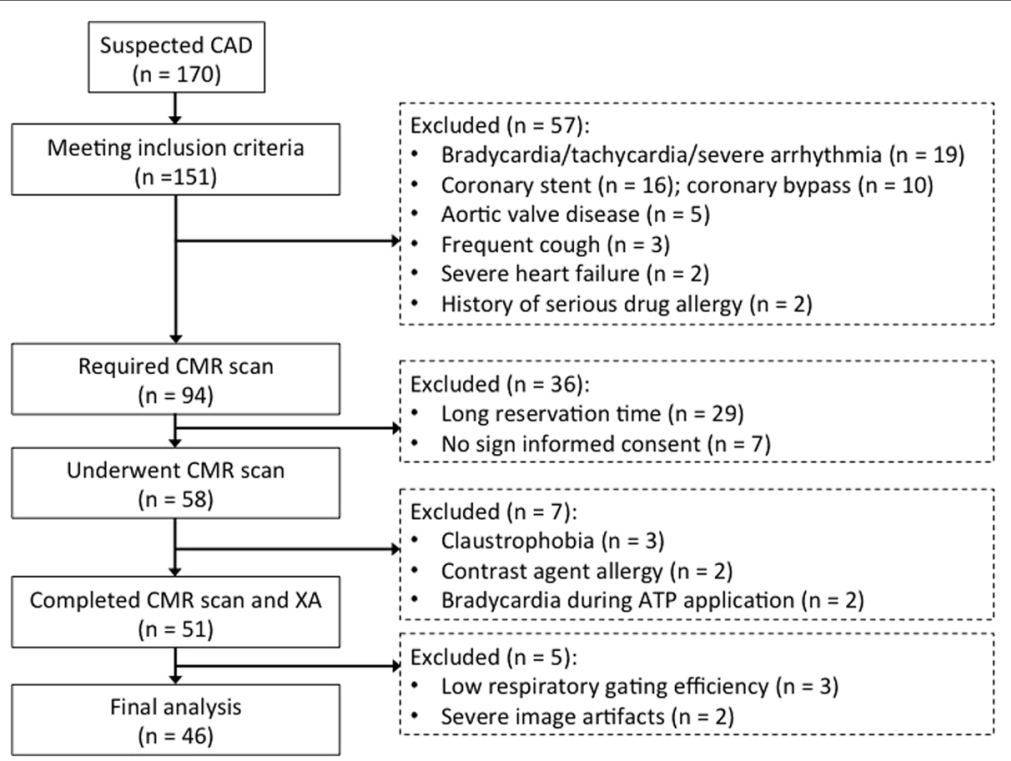

Fig. 1 Study flow chart and reasons for exclusion of patients 


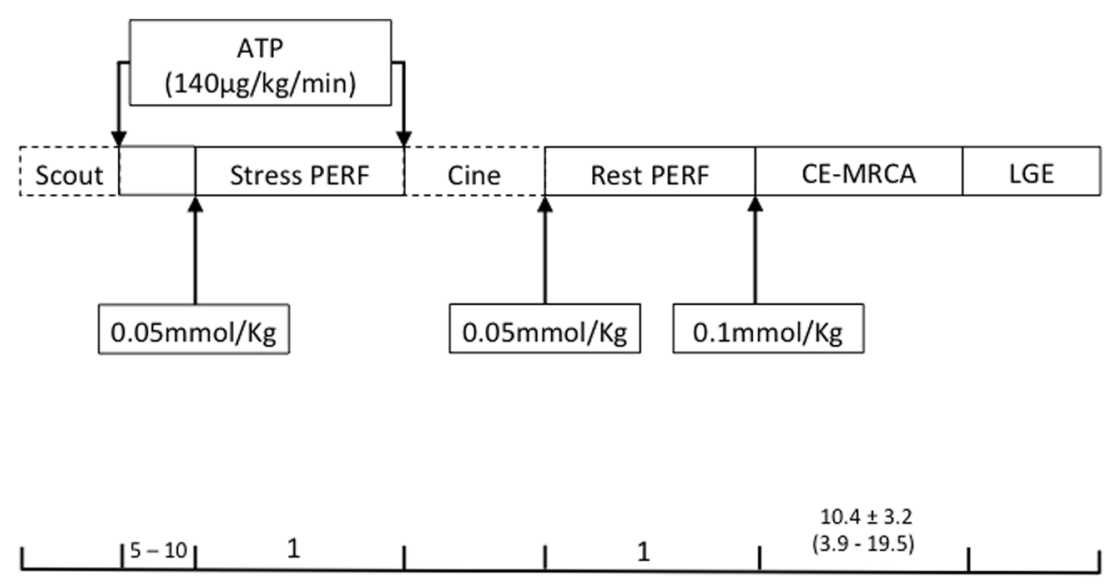

$(\min )$

Fig. 2 CMR Protocol

images were assessed on a per-segment basis. The 15segment American Heart Association (AHA) classification system was used for CAD diagnosis. Examples of various CE-MRCA image qualities (ImQ) are shown in Fig. 3. Images with ImQ 2 to 4 were evaluated for stenosis, and ImQ 1 was deemed non-assessable. CE-MRCA at each segment was graded on 5 -point scale: $0=$ normal; $1=$ mild stenosis $(<50 \%) ; 2=$ significant stenosis $\geq 50 \%$; 3 = uninterpretable (cannot exclude significant stenosis); $4=$ not visible. Segments with grades 0 and 1 were classified as negative and those with grade 2 were classified as positive. Segments with grades 3 and 4 were classified as negatives or positive depending on the results of MPI or LGE. When combining CE-MRCA and MPI or LGE, if any of the tests was positive, the overall result was deemed positive; if all were negative, the overall result was negative.

CMR perfusion images were assessed by visual comparison of stress and rest scans (17 segment American Heart Association (AHA) model, excluding the apex [14]). A perfusion deficit was determined if there were ten or more consecutive frames of apparent signal reduction. LGE images were analyzed visually using AHA segmentation, and bright segments were classified as positive.
Patients underwent x-ray coronary angiography (XA) within 1 week after CMR. The 15-segment AHA classification system was used for CAD diagnosis. Stenoses were quantitatively evaluated for segments with a diameter of $>$ $2 \mathrm{~mm}$ (QuantCor software, QCA, Siemens Healthineers). Functionally significant CAD was defined as the presence of luminal stenosis $\geq 50 \%$. Correspondence between LV myocardial segments and coronary arteries was determined based on the AHA model [14].

\section{Statistical analysis}

Continuous data are reported as mean \pm standard deviation. Data analysis was performed on both patient- and vesselbases. Sensitivity and specificity were calculated using true positive (TP), true negative (TN), false positive (FP), and false negative (FN) rates using XA as reference. The $\mathrm{McNe}$ mar test was used to calculate differences between proportions (sensitivity, specificity, and accuracy) obtained from paired observations. Receiver operating characteristic (ROC) curves were calculated to assess the diagnostic efficacy and the area under the ROC curves (AUC) were compared between different imaging methods. Cohen's kappa statistic was used to assess inter-observer agreements. SPSS

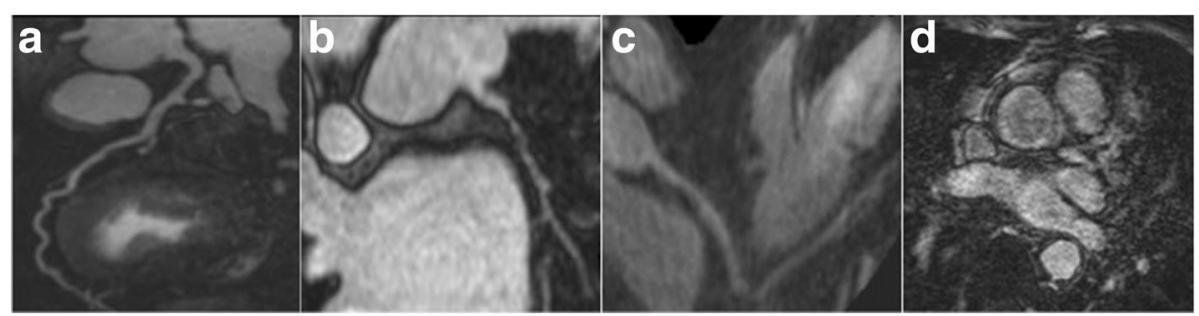

Fig. 3 Examples of contrast enhanced magnetic resonance coronary angiography (CE-MRCA) imaging quality grades, Grade $4=$ minimal artifacts, excellent signal and contrast on left anterior descending coronary artery (LAD) (a); Grade $3=$ slight artifacts and good signal and contrast on the left circumflex coronary artery $(\mathrm{LCX})(\mathbf{b})$; Grade $2=$ moderate artifacts and fair signal and contrast on the right coronary artery $(\mathrm{RCA})(\mathbf{c})$; Grade $1=$ severe image artifacts and poor signal and contrast on $\operatorname{LAD}(\mathbf{d})$ 
statistics (version 21, International Business Machines, Armonk, New York, USA) was used for data analysis. $P<0$. 05 was considered a significant difference.

\section{Results}

One hundred and fifty one patients met the inclusion criteria of which 51 underwent CMR scanning. Detailed exclusion reasons were summarized in Fig. 1. The mean exam duration was $58.0 \pm 7.9 \mathrm{~min}$. The final analysis included 46 individuals $(54.0 \pm 11.5$ years, $71.7 \%$ males $)$ after excluding incomplete CE-MRCA $(n=5)$. Patient characteristics are shown in Table 1.

\section{Image quality and assessability of MRCA}

A total of $90.2 \%(46 / 51)$ of CE-MRCA studies were included in data analysis. Five patients were excluded: CEMRCA was aborted in three patients due to extremely low respiratory gating efficiency (navigator efficiency $<20 \%$ by the time half of the imaging data were collected); severe motion artifacts were present in two patients as evaluated by an experienced radiologist. The mean scan time was $10.4 \pm 3.2 \mathrm{~min}$, ranging from 3.9 to $19.5 \mathrm{~min}$. Patients did not receive medicine to reduce their heart rate (HR), thus 22 patients had a HR > $65 \mathrm{bpm}$ and 24 patients had $\mathrm{HR} \leq$ $65 \mathrm{bpm}$, with a mean HR of $67.3 \pm 9.6 \mathrm{bpm}$. The average navigator respiratory gating efficiency was $35.0 \pm 9.1 \%$. CE-MRCA was acquired during diastole in 21 patients (77\% with $\mathrm{HR} \leq 65 \mathrm{bpm})$ and during systole in $25(67 \%$ with $\mathrm{HR}>65 \mathrm{bpm})$. The average scan time of patients with diastolic acquisition was similar to that of systolic acquisition $(10.3 \pm 2.6 \mathrm{~min}$ vs. $10.4 \pm 3.8 \mathrm{~min}, p=0.28)$.

Significant stenosis was found in $73.9 \%$ patients $(34 / 46)$ based on MRCA. The kappa value for inter-observer agreement on per-patient basis for the identification of

Table 1 Characteristics of the study population

\begin{tabular}{|c|c|c|c|}
\hline & Sensitivity (\%) & Specificity (\%) & Accuracy (\%) \\
\hline \multicolumn{4}{|c|}{ Patient Basis ( $N=46$, CAD 73.9\%) } \\
\hline MPI & $76.5(26 / 34)$ & $66.7(8 / 12)$ & $73.9(34 / 46)$ \\
\hline MPI/LGE & $76.5(26 / 34)$ & $66.7(8 / 12)$ & $73.9(34 / 46)$ \\
\hline MRCA & $97.1(33 / 34)$ & $91.7(11 / 12)$ & $95.7(44 / 46)$ \\
\hline CE-MRCA+MPI/LGE & $100(34 / 34)$ & $58.3(7 / 12)$ & $89.1(41 / 46)$ \\
\hline LGE & $41.2(14 / 34)$ & $91.7(11 / 12)$ & $54.3(25 / 46)$ \\
\hline CE-MRCA+LGE & $97.1(33 / 34)$ & $83.3(10 / 12)$ & $93.5(43 / 46)$ \\
\hline \multicolumn{4}{|c|}{ Vessel Basis ( $N=138$, CAD 30.4\%) } \\
\hline MPI & $67.9(38 / 56)$ & $82.9(68 / 82)$ & $76.8(106 / 138)$ \\
\hline $\mathrm{MPI} / \mathrm{LGE}$ & $67.9(38 / 56)$ & $82.9(68 / 82)$ & $76.8(106 / 138)$ \\
\hline CE-MRCA & $89.3(50 / 56)$ & $89.0(73 / 82)$ & $89.1(123 / 138)$ \\
\hline CE-MRCA+MPI/LGE & $94.6(53 / 56)$ & $73.2(60 / 82)$ & $81.9113 / 138)$ \\
\hline LGE & $28.6(16 / 56)$ & $95.1(78 / 82)$ & $68.1(94 / 138)$ \\
\hline CE-MRCA+LGE & $89.3(50 / 56)$ & $84.1(69 / 82)$ & $86.2(119 / 138)$ \\
\hline
\end{tabular}

significant stenosis was 0.78 (95\% CI, 0.55-1.0). Of the 34 patients, 16 (47.1\%) had 1-vessel CAD, 11 (32.4\%) had 2vessel CAD, and 7 (20.6\%) had 3-vessel CAD. On pervessel basis, 42.8\% (59/138) vessels had significant stenosis detected by MRCA, including 30.5\% right coronary artery (RCA) (18/59), 40.7\% left anterior descending artery (LAD) (24/59), and $28.8 \%$ left circumflex artery (LCX) (17/59).

Of the total 690 segments, 529 segments had diameter $>2 \mathrm{~mm}$ and $96.4 \%(510 / 529)$ were judged to have adequate image quality (ImQ $4=391$, ImQ $3=101$, ImQ 2 $=18$ ) with an average ImQ of $3.7 \pm 0.5$. For stenosis grade, of 510 segments, 410 (80.4\%) had grade 0 or 1,89 (17.5\%) had grade 2 . Eleven segments had grades 3 (uninterpretable) or 4 (invisible).

On per-patient basis, CE-MRCA had a sensitivity of $97.1 \%$, specificity of $91.7 \%$, accuracy of $95.7 \%$, and AUC of 0.94 (95\% CI, 0.83-0.99). On per-vessel basis, CEMRCA had a sensitivity of $89.3 \%$, specificity of $89.0 \%$, accuracy of $89.1 \%$, and AUC of 0.89 (95\% CI, 0.83-0.94).

\section{Combination of CE-MRCA with MPI}

The mean heart rates were $91 \pm 11.4 \mathrm{bpm}$ (ranging from 70 to $120 \mathrm{bpm}$ ) during stress perfusion and $67 \pm 9.5 \mathrm{bpm}$ (ranging from 50 to $86 \mathrm{bpm}$ ) during rest perfusion. The average increased HR was $23 \pm 8.5 \mathrm{bpm}$ (ranging from 9 to $42 \mathrm{bpm}$ ). On per-patient basis, the kappa value for inter-observer agreement for the identification of perfusion defect was 0.73 (95\% CI, 0.52-0.93).

Of the total 736 segments in the 46 patients, $23.2 \%$ (171/736) was positive on MPI alone, corresponding to 52 main branches (RCA 21, LAD 16, and LCX 15). After adding CE-MRCA, the positive results increased to 75 main branches (RCA 25, LAD 29, and LCX 21).

The diagnostic performance of combined CE-MRCA and MPI compared to MPI alone was assessed in 46 patients and 138 vessels respectively. Results are given in Table 2. Compared to MPI alone, adding CE-MRCA to MPI showed higher sensitivity $(100 \%$ vs. $76.5 \%, p<0.01)$, similar specificity $(58.3 \%$ vs. $66.7 \%, p=0.6)$, and higher accuracy (89.1\% vs. $73.9 \%, \mathrm{p}<0.01)$ on per-patient basis (Fig. 4). ROC analysis showed that there was no significant difference between the combination of CE-MRCA and MPI (AUC $=0.79 ; 95 \%$ CI, 0.65-0.90), compared with MPI alone $(\mathrm{AUC}=0.72 ; 95 \% \mathrm{CI}, 0.56-0.84)(p=0.17)$. AUC was improved in vessel-based analysis $(0.84$ [95\% CI, 0.77-0.90] vs. 0.75 [95\% CI, 0.67-0.82], $p=0.01$ ) (Fig. 5).

In MPI alone, 4 patients were FPs and 8 patients were FNs. By adding CE-MRCA, the number of FPs was increased by 1 and number of FNs was decreased from 8 to 0 (Fig. 6).

\section{Combination of MRCA with LGE}

Based on LGE alone, 15 patients were founded positive and 31 patients were negative. Adding CE-MRCA to LGE, 
Table 2 Diagnostic accuracy of the different imaging methods and their combination on per-patient and per-vessel bases

\begin{tabular}{ll}
\hline Number of patients $(\mathrm{n})$ & 46 \\
Male sex & $33(71.7 \%)$ \\
Age $(\mathrm{yrs})$ & $54 \pm 11.5$ \\
Body-mass index $\left(\mathrm{kg} / \mathrm{m}^{2}\right)$ & $26 \pm 3.2$ \\
Hypercholesterolemia & $13(28.2 \%)$ \\
Hypertension & $29(63.0 \%)$ \\
Diabetes mellitus & $8(17.4 \%)$ \\
Positive smoking history & $17(37.0 \%)$ \\
Current smoker & $16(34.8 \%)$ \\
Family history of CAD & $6(13.0 \%)$ \\
\hline
\end{tabular}

$C A D$ coronary artery disease, $M P I$ stress-rest myocardial perfusion imaging, $L G E$ late gadolinium enhancement, CE-MRCA contrast enhanced magnetic resonance coronary angiography

35 patients were founded positive and 11 patients were negative. Although the number of FPs was slightly increased (from 1 to 2), the number of FNs was dramatically decreased from 20 to 1, compared to LGE alone (Fig. 6).

Adding CE-MRCA to LGE showed significantly higher sensitivity $(97.1 \%$ vs. $41.2 \%, p<0.01)$ translating into better diagnostic performance (AUC $=0.90$ [95\% CI, 0.78-0.97] vs. 0.66 [95\% CI, 0.51-0.80], $p<$ 0.01 ) (Fig. 7). Although specificity was inferior $(83.3 \%$ vs. $91.7 \%, p=0.02)$, diagnostic accuracy was significantly increased from $54.3 \%$ to $93.5 \%$, compared to LGE alone (Fig. 4). The same trends were also found in vessel-based analysis (Table 2).

\section{Combination of CE-MRCA with MPI/LGE}

In the 46 patients, $23.2 \%(171 / 736)$ was positive on MPI/LGE. The diagnostic performance for the combination of CE-MRCA and MPI/LGE compared to MPI/ LGE are given in Table 2. Compared to MPI/LGE alone, adding CE-MRCA showed higher sensitivity (100\% vs. $76.5 \%, p<0.01)$ and similar specificity $(58.3 \%$ vs. $66.7 \%$, $p=0.6)$. Overall accuracy was improved from $73.9 \%$ to $89.1 \%$ on per-patient basis (Fig. 4). ROC analysis showed that there was no significant difference between the combined CE-MRCA and MPI/LGE (AUC $=0.79$; 95\% CI, 0.65-0.90), compared with MPI/LGE (AUC =0.72; 95\% CI, 0.56-0.84) $(p=0.17)$. AUC was improved on per-vessel basis $(0.84$ [95\% CI, 0.77-0.90] vs. 0.75 [95\% CI, 0.67-0.82], $p=0.01$ ) (Fig. 5).

It should be pointed out that these results were the same as that of MPI/LGE compared to MPI alone. A positive diagnosis is obtained when either of MPI or LGE is positive. Positive LGE in comparison to negative MPI may result from small subendocardial infarcts. Apparently it was not the case in this patient population.

\section{Discussion}

To our knowledge, this is the first study to evaluate the additive value of $3 \mathrm{D}$ whole-heart CE MRCA to conventional CMR MPI and LGE for detecting CAD on a $3 \mathrm{~T}$ CMR system. We demonstrated that the integration of CE-MRCA into a comprehensive stress-rest MPI and LGE protocol significantly improved sensitivity and diagnostic accuracy. These results conflict with the previous

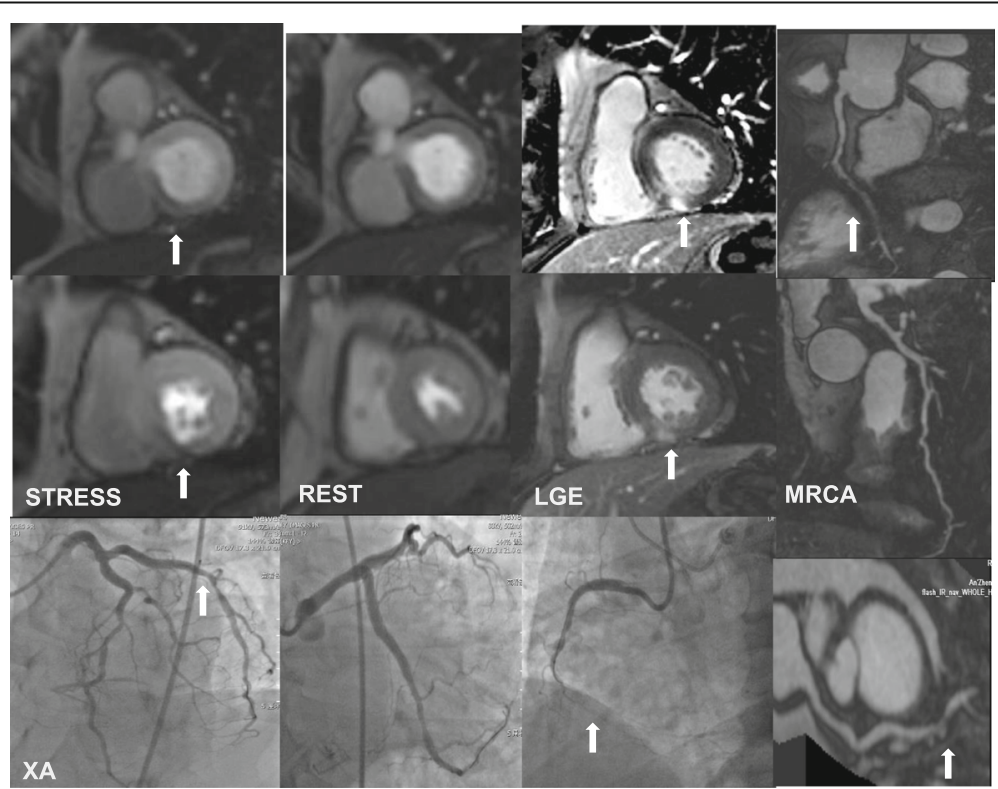

Fig. 4 One case illustrating myocardial perfusion imaging (MPI), late gadolinium enhancement (LGE), CE-MRCA and x-ray angiography (XA) angiographic findings in patient with coronary artery disease (CAD), Male/60 yrs. MPI (+) + MRCA $(+)=$ combination $(+)$. LGE $(+)+\mathrm{MRCA}(+)=$ combination $(+)$. MPI/LGE $(+)+$ MRCA $(+)=$ combination $(+)$. Corresponding XA shows significant RCA and LCX stenoses 


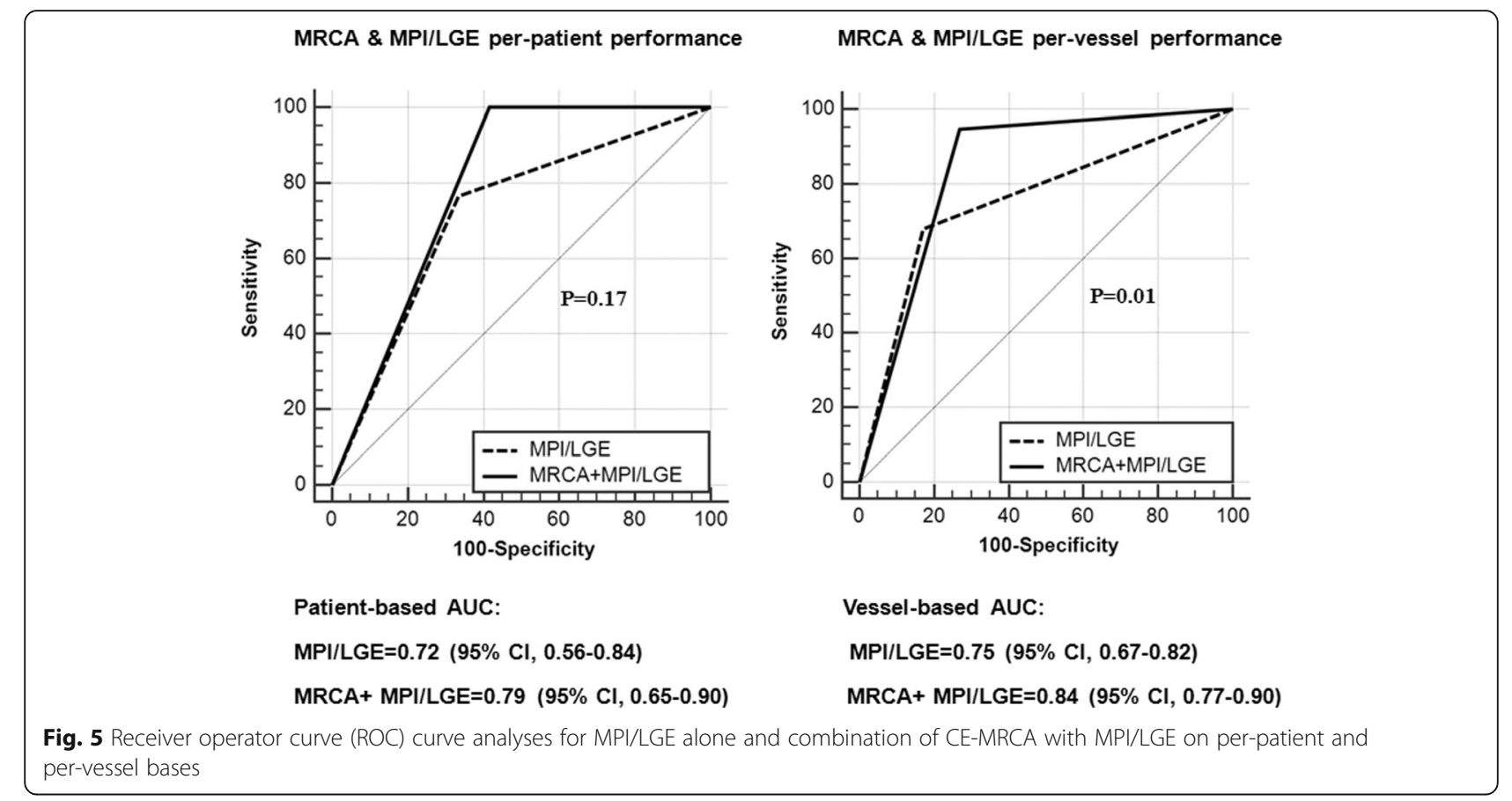

study at 1.5 T [15], in which MRCA did not increase the overall diagnostic accuracy. Possible reasons include the enhanced contrast between blood and background and increased image quality due to contrast enhanced imaging and shorter scan time on $3 \mathrm{~T}$ as compared to noncontrast-enhanced imaging at $1.5 \mathrm{~T}$. CE-MRCA increased the number of assessable coronary artery segments in comparison to unenhanced MRCA [16], especially distal segments [17]. Sommer et al. [18] and Bi et al. [19] demonstrated a significant increase in SNR from 1.5 to 3.0 T. Liu et al. [20] and Prompona et al. [21] found significant increase in contrast-to-noise ratio at 3 .

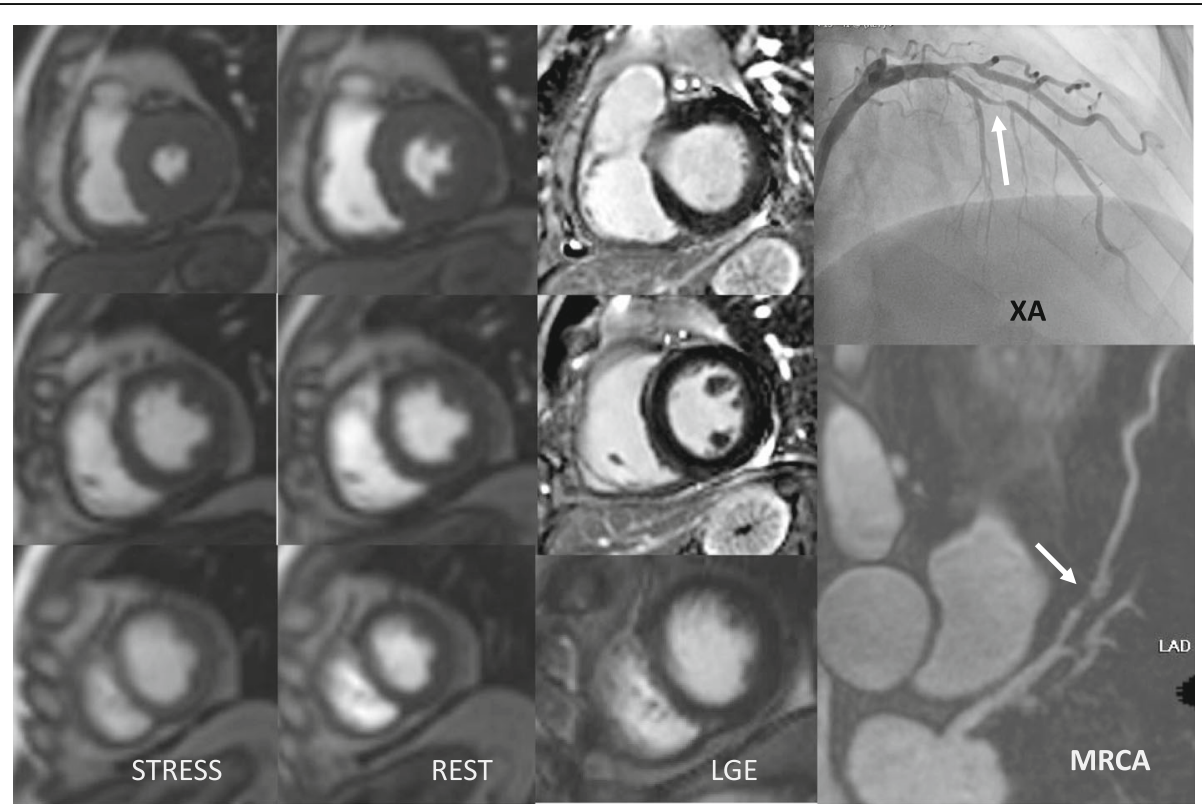

Fig. 6 One case illustrating MPI, LGE, CE-MRCA and XA angiographic findings in patient with CAD. Male/42 yrs. MPI $(-)+$ CE-MRCA $(+)=$ combination $(+)$. LGE $(-)+$ CE-MRCA $(+)=$ combination (+). MPI/LGE $(-)+$ CE-MRCA $(+)=$ combination $(+)$. Corresponding XA shows significant LAD stenosis, correctly identified by CE-MRCA. 
MRCA \& LGE per-patient performance

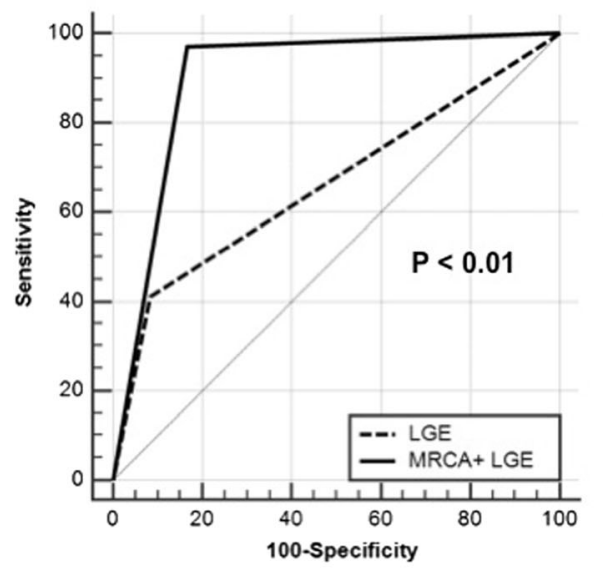

Patient-based AUC:

LGE $=0.66(95 \% \mathrm{Cl}, 0.51-0.80)$

MRCA+ LGE $=0.90(95 \% \mathrm{Cl}, 0.78-0.97)$
MRCA \& LGE per-vessel performance

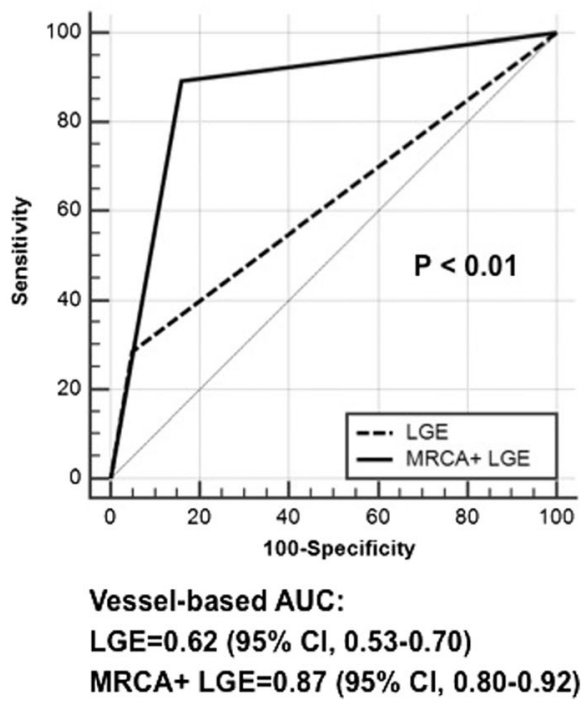

Fig. 7 ROC curve analysis for LGE alone and combination of CE-MRCA with LGE on per-patient and per-vessel bases

$0 \mathrm{~T}$ as compared to $1.5 \mathrm{~T}$. Indeed, $19 \%$ of MRCA at 1. $5 \mathrm{~T}$ had poor image quality [15] as compared to $3.6 \%$ in this study at $3 \mathrm{~T}$.

Another difference between this study and the previous studies at $1.5 \mathrm{~T}$ is the definition of significant stenosis. In this study, significant stenosis was defined as luminal narrowing of $\geq 50 \%$ as determined on $\mathrm{XA}$, while in previous studies, significant stenosis was defined as luminal narrowing of $\geq 90 \%$ stenosis or fractional flow reserve (FFR) $\leq$ 0.80 by Bettencourt $\mathrm{N}$ et al. [15]; or luminal narrowing of $\geq 70 \%$ by Ripley DR et al. [22].

In this study, adding CE-MRCA to MPI and LGE reduced the incidents of false negative (from 8 to 0 ). These 8 false negative patients were confirmed by coronary angiography as having $50-70 \%$ stenosis $(n=4), 70-80 \%$ stenosis $(n=2)$, chronic total occlusion with good collateral circulation $(n=2)$. CE-MRCA accurately identified these stenoses without myocardial ischemia or infarction, thereby reducing false negatives and improving the sensitivity of diagnosis. By adding CE-MRCA to LGE, 19 of the 20 false negatives were correctly depicted by CE-MRCA.

In this study, there were 4 false-positives using MPI. Among the four patients, three had microvascular coronary dysfunction patients, showing extensive annular ischemia in the endocardium but no coronary artery stenosis on XA.

Therefore, although the detection of the morphologically significant coronary stenosis and that of hemodynamically significant coronary stenosis are clinically different, but this study shows that CE-MRCA can detect the morphologically significant coronary stenosis in patients without hemodynamic significant stenosis, so as to avoid the omission of severe stenosis, so that patients can get the corresponding clinical treatment as early as possible; and CE-MRCA can screened out the patients without three main coronary artery morphologically stenosis but with hemodynamic stenosis, so that these patients can avoid invasive examination.

\section{Study limitations}

In this study, $10 \%$ patients excluded due to CE-MRCA imaging issues, 3/51 patients were not able to complete CE-MRCA due to low respiratory gating efficiency and $2 / 51$ patients had severe motion artifacts. They were not included in data analysis. $26 \%$ of CE-MRCA segments were not analysable for small diameter $(161 / 690)$ and severe image artifacts and poor signal and contrast (19/ 690). This is a single center study with very small patient numbers. A prospective, multicenter study with a larger sample size is needed to further confirm the findings of the study.

\section{Conclusion}

On $3 \mathrm{~T}$, integration of successful 3D whole-heart CEMRCA into a comprehensive stress-rest myocardial perfusion or LGE protocol significantly improved sensitivity and diagnostic accuracy for detection of CAD.

\section{Abbreviations}

AHA: American Heart Association; AUC: The area under the ROC curves; CAD: Coronary artery disease; CE-MRCA: Contrast-enhanced magnetic resonance coronary angiography; CMR: Cardiovascular magnetic resonance; ECG: Electrocardiogram; FFR: Fractional flow reserve; FN: False negative; FP: False positive; HR: Heart rate; ImQ: Image quality; LAD: Left anterior descending coronary artery; LCX: Left circumflex coronary artery; LGE: Late gadolinium enhancement; LV: Left ventricle/left ventricular; MPI: Stress/rest 
myocardial perfusion imaging; RCA: Right coronary artery.; ROC: Receiver operating characteristic; TI: Inversion time; TN: True negative; TP: True positive; XA: X-ray coronary angiography

\section{Acknowledgements}

We thank Xiantao Song and his team for their assistance. We are particularly grateful for the assistance given by Debiao Li.

\section{Availability of data and materials}

The data sets during and/or analysed during the current study available from the corresponding author on reasonable request.

\section{Authors' contributions}

LZ made substantial contributions to the acquisition and interpretation of data for the work and to drafting the manuscript. ZF and DL made substantial contributions to the conception and design of the work, the acquisition, analysis, and interpretation of data for the work and to drafting the work and revising it critically for important intellectual content. XS, JL, RD and LD made substantial contributions to the acquisition and interpretation of data for the work and to revising it critically for important intellectual content. JA made substantial contributions to the design and improvement of MR scanning sequences. All authors provided final approval of the version to be published.

\section{Ethics approval and consent to participate}

The study protocol was approved by the Ethics Committee of Beijing Anzhen Hospital. Written informed consent was obtained from the participants for publication of their individual details and images in this manuscript. The consent forms are held at the authors' institution and are available for review by the Editor-in-Chief.

\section{Consent for publication}

All the subjects provided written informed consent for the publication of accompanying images in this manuscript. The consent forms are held in the authors' institution and are available for review by the Editor-in-Chief.

\section{Competing interests}

The authors declare that they have no competing interests.

\section{Publisher's Note}

Springer Nature remains neutral with regard to jurisdictional claims in published maps and institutional affiliations.

\section{Author details}

'Department of Radiology, Beijing Anzhen Hospital, Capital Medical University, Anzhenli Avenue, Chao Yang District, Beijing 100029, China. 2Department of Cardiology, Beijing Anzhen Hospital, Capital Medical University, Beijing, China. ${ }^{3}$ Siemens Shenzhen Magnetic Resonance Ltd, Guangdong Shenzhen, China. “Biomedical Imaging Research Institute, Cedars-Sinai Medical Center, University of California, Los Angeles, USA.

\section{Received: 23 June 2017 Accepted: 6 April 2018}

\section{Published online: 30 April 2018}

\section{References}

1. Murray CJ, Lopez AD. Global mortality, disability, and the contribution of risk factors: global burden of disease study. Lancet. 1997;349(9063):1436-42.

2. Lozano R, Naghavi M, Foreman K, Lim S, Shibuya K, Aboyans V, et al. Global and regional mortality from 235 causes of death for 20 age groups in 1990 and 2010: a systematic analysis for the global burden of disease study 2010. Lancet. 2012;380(9859):2095-128.

3. Pakkal M, Raj V, Mccann GP. Non-invasive imaging in coronary artery disease including anatomical and functional evaluation of ischaemia and viability assessment. Br J Radiol. 2011;84(Spec Iss 3):S280-95.

4. Rocha-Filho JA, Blankstein R, Shturman LD, Bezerra HG, Okada DR, Rogers IS, et al. Incremental value of adenosine induced stress myocardial perfusion imaging with dual-source ct at cardiac ct angiography. Radiology. 2010;254(2):410-9.

5. Pontone G, Andreini D, Baggiano A, Bertella E, Mushtaq S, Conte E, et al. Functional relevance of coronary artery disease by cardiac magnetic resonance and cardiac computed tomography: myocardial perfusion and fractional flow reserve. Biomed Res Int. 2015;2015:297696.
6. Pilz G, Patel PA, Fell U, Ladapo JA, Rizzo JA, Fang H, et al. Adenosine-stress cardiac magnetic resonance imaging in suspected coronary artery disease: a net cost analysis and reimbursement implications. Int J Cardiovasc Imaging. 2011;27:113-21.

7. He Y, Pang J, Dai Q, Fan Z, An J, Li D. Diagnostic performance of self-navigated whole-heart contrast-enhanced coronary 3-T MR angiography. Radiology. 2016;281(2):401-8

8. Sakuma H, Ichikawa Y, Chino S, Hirano T, Makino K, Takeda K. Detection of coronary artery. stenosis with whole-heart coronary magnetic resonance angiography. J Am Coll Cardiol. 2006;48(10):1946-50.

9. Kato S, Kitagawa K, Ishida N, Ishida M, Nagata M, Ichikawa Y, et al. Assessment of coronary artery disease using magnetic resonance coronary angiography: a national multicenter trial. J Am Coll Cardiol. 2010;56(12):983-91.

10. Yang Q, Li K, Liu X, Bi X, Liu Z, An J, et al. Contrast-enhanced whole-heart coronary magnetic resonance angiography at 3.0-T: a comparative study with X-ray angiography in a single center. J Am Coll Cardiol. 2009:54(1):69-76.

11. Nagata M, Kato S, Kitagawa K, Ishida N, Nakajima H, Nakamori S, et al. Diagnostic accuracy of 1.5-T unenhanced whole-heart coronary MR angiography performed with 32-channel cardiac coils: initial single-center experience. Radiology. 2011;259(2):384-92.

12. Heer T, Reiter S, Höfling B, Pilz G. Diagnostic performance of non-contrastenhanced whole-heart magnetic resonance coronary angiography in combination with adenosine stress perfusion cardiac magnetic resonance imaging. Am Heart J. 2013;166(6):999-1009.

13. Klein C, Gebker R, Kokocinski T, Dreysse S, Schnackenburg B, Fleck E, et al. Combined magnetic resonance coronary artery imaging, myocardial perfusion and late gadolinium enhancement in patients with suspected coronary artery disease. J Cardiovasc Magn Reson. 2008;10:45.

14. Cerqueira MD, Weissman NJ, Dilsizian V, Jacobs AK, Kaul S, Laskey WK, et al. Standardized myocardial segmentation and nomenclature for tomographic imaging of the heart. A statement for healthcare professionals from the cardiac imaging Committee of the Council on clinical cardiology of the American Heart Association. Circulation. 2002;105(4):539-42.

15. Bettencourt N, Ferreira N, Chiribiri A, Schuster A, Sampaio F, Santos L, et al. Additive value of magnetic resonance coronary angiography in a comprehensive cardiacmagnetic resonance stress-rest protocol for detection of functionally significant coronary artery disease: a pilot study. Circ Cardiovasc Imaging. 2013:6(5):730-8.

16. Wagner M, Rösler R, Lembcke A, Butler C, Dewey M, Laule M, et al. Whole-heart coronary magnetic resonance angiography at 1.5 Tesla: does a blood-pool contrast agent improve diagnostic accuracy? Investig Radiol. 2011;46(3):152-9.

17. Yang Q, Li K, Liu X, Du X, Bi X, Huang F, et al. 3.0T whole-heart coronary magnetic resonance angiography performed with 32-channel cardiac coils: a single-center experience. Circ Cardiovasc Imaging. 2012;5:573-9.

18. Sommer T, Hackenbroch $M$, Hofer U, Schmiedel A, Willinek W, Flacke S, et al. Coronary MR angiography at 3.0 T versus that at $1.5 \mathrm{~T}$ : initial results in patients suspected of having coronary artery disease. Radiology. 2005;234:718-25.

19. Bi X, Li D. Coronary arteries at 3.0 T: contrast-enhanced magnetizationprepared three-dimensional breathhold MR angiography. J Magn Reson Imaging. 2005;21:133-9.

20. Liu X, Bi X, Huang J, Jerecic R, Carr J, Li D. Contrast-enhanced whole-heart coronary magnetic resonance angiography at $3.0 \mathrm{~T}$ : comparison with steadystate free precession technique at 1.5 T. Investig Radiol. 2008;43:663-8.

21. Prompona M, Cyran C, Nikolaou K, Bauner K, Reiser M, Huber A. Contrastenhanced whole-heart MR coronary angiography at 3.0 T using the intravascular contrast agent Gadofosveset. Investig Radiol. 2009;44(7):369-74.

22. Ripley DP, Motwani M, Brown JM, Nixon J, Everett CC, Bijsterveld P, et al. Individual component analysis of the multi-parametric cardiovascular magnetic resonance protocol in the CE-MARC trial. J Cardiovasc Magn Reson. 2015;17:59. 